\title{
SEIZURE PRESENTING WITH FRACTURE AT ONSET -A CASE REPORT
}

\author{
O.P. Lekhra, A. Ahuja
}

1. Associate Professor, Department of Neurology, Sri Aurobindo Medical College \& PG Institute, Indore.

2. Associate Professor, Department of Radio Diagnosis, Sri Aurobindo Medical College \& PG Institute, Indore.

\section{CORRESPONDING AUTHOR}

Dr. O.P. Lekhra

Quince-205,

Shalimar Township,

AB Road, INDORE (MP),

E-mail: olekhra@yahoo.com,

Ph: 00919893185522

INTRODUCTION: Injuries are very common after a convulsive episode of seizure. The patient can sustain soft tissues injuries in the form of tongue bite, and cuts or abrasions over skin. But more problematic are bony injuries like dislocation and fracture ${ }^{1}$. These injuries can be primarily due to epileptic fit or secondary to the accidental fall, which the patient sustain after the seizure ${ }^{1}$. We describe a young male who had bilateral posterior dislocation of shoulder in the first episode of seizure.

KEY WORDS: Seizure, Dislocation, Fracture

CASE REPORT: A 32 year male had two episodes of serial generalised tonic clonic convulsions in succession. There was no history of fever / chronic headache /visual disturbances/trauma. On examination he was conscious and oriented well in time and person . BP 132/80 mmHg right upper limb in supine position, pulse rate $88 /$ minute and regular. Both eyes fundus were normal. Both the shoulder joints were tender and range of movements was also restricted. Rest of the neurovascular examination was unremarkable except that range of movements were restricted in both shoulder joints. Fullness, cellulites and tenderness was present over right shoulder. Investigations revealed routine blood and biochemistry to be normal. CT Brain - normal. EEG showed generalised spike and wave discharges. X-ray both shoulder joints A/P view revealed posterior dislocation. Left humerous had simple fracture anatomical neck (Fig 1a) but right humerous also had comminuted fracture anatomical neck (Fig1b). Patient underwent needful surgical intervention. He was put on two anticonvulsants considering the severity of the injury, he sustained in the first seizure itself.

DISCUSSION: Epilepsy is a common problem in the general population. About three percent of seizure patients are injured directly due to the convulsive event or indirectly due to sudden fall following the seizure ${ }^{2}$. Multiple bony fractures and dislocations have been described following seizures .Posterior dislocation of the shoulder joint is the most common type of dislocation associated with seizure, though anterior dislocation has been occasionally described ${ }^{3}$.The fracture dislocation of the humerous is typically associated with epilepsy, electrocution or extreme trauma. The shoulder joint is commonly predisposed for injury during a convulsive seizure because of the shallow glenoid cavity and the typical position of adduction, internal rotation and flexion of it during the convulsion. With the intense spasm of the muscles the humeral head is forced superiorly and posteriorly over the glenoid cavity. Thus the humeral head stays locked behind the glenoid. Then with further convulsive force, the humeral head 
impinged against the glenoid rim, resulting eventually in a complex proximal humeral fracture. Next common are the bony fractures of neck of femur in seizure.They are usually bilateral and in rare cases acetabular fracture also can be seen with seizure ${ }^{4}$.The tremendous forceful contractions of pelvitrochanteric muscles acting in a craniomedial direction in a seizure can lead to fracture dislocation of the hip joint. Compression fracture of the vertebrae is the next in order in epileptics. The incidence of symptomatic spinal fracture from a seizure is rare, whereas asymptomatic spinal fracture may be as high as fifteen percent ${ }^{5}$. The most common fracture location in the vertebral column is the upper to mid-thoracic region. Here also forceful contractions of the axial musculature in a generalized tonic clonic seizure produce hyperflexion of spine leading to fractures, especially of the thoracic vertebra6. To diagnose compression fractures of the spine, index of suspicion should be high in the patient presenting with complaints of ill-localized back pain without any history of trauma or fall. Other potential sites of fracture-dislocations in seizure are facial bones, proximal humerous, distal radius and the ankle bones? .

This case demonstrates that bilateral posterior shoulder dislocations are implicated with unwitnessed seizures. Therefore, a high index of suspicion of a seizure disorder must exist in situations where patient wakes up with bony fracture or dislocation with no history of trauma.

\section{REFERENCES:}

1. Finelli FP and Cardi JK.Seizure as a cause of fracture. Neurology 1989; 39: 858-860

2. Souverein PC,Webb DJ, Petri H, Weil J, Van Staa TP and EgbertsT. Incidence of fractures among epilepsy patients: a population-based retrospective cohort study in the general practice research database.Epilepsia.2005;46(2): 304-310.

3. Sadhra K. Unusual dislocations associated with epileptic fits. British Medical Journal 1984; 288: 681

4. Gissane W, Blair D, Rank BK. Fractures of the Neck of the Femur in Convulsion Therapy. Lancet 1940; 1: 450-3

5. Pedersen KK, Christiansen C, Ahlgren P, Lund M. Incidence of fractures of the vertebral spine in epileptic patients. Acta Neurol Scand. 1976; 54 (2):200-203.

6. 6 Vasconcelos D. Compression fractures of the vertebrae during major epileptic seizures. Epilepsia 1973; 14:323-328.

7. Gosens T,Poels PJ,Rondhuis JJ. Posterior dislocation fractures of the shoulder in seizure disorders--two case reports and a review of literature. Seizure 2000; 9(6):446-8 


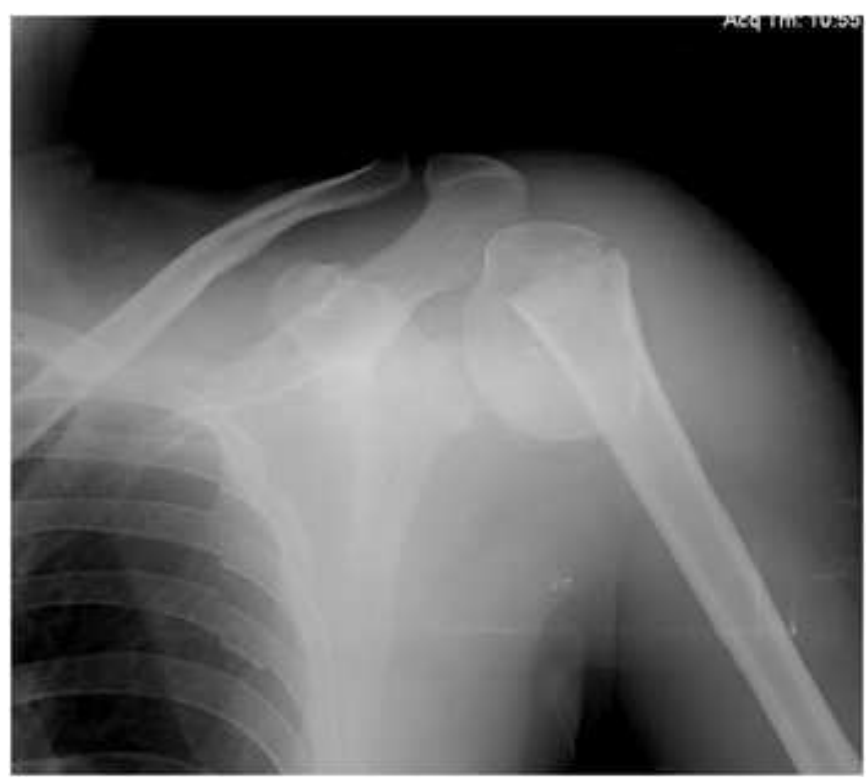

Fig 1a. Fracture anatomical neck of the left humerous and posterior dislocation of the shoulder.

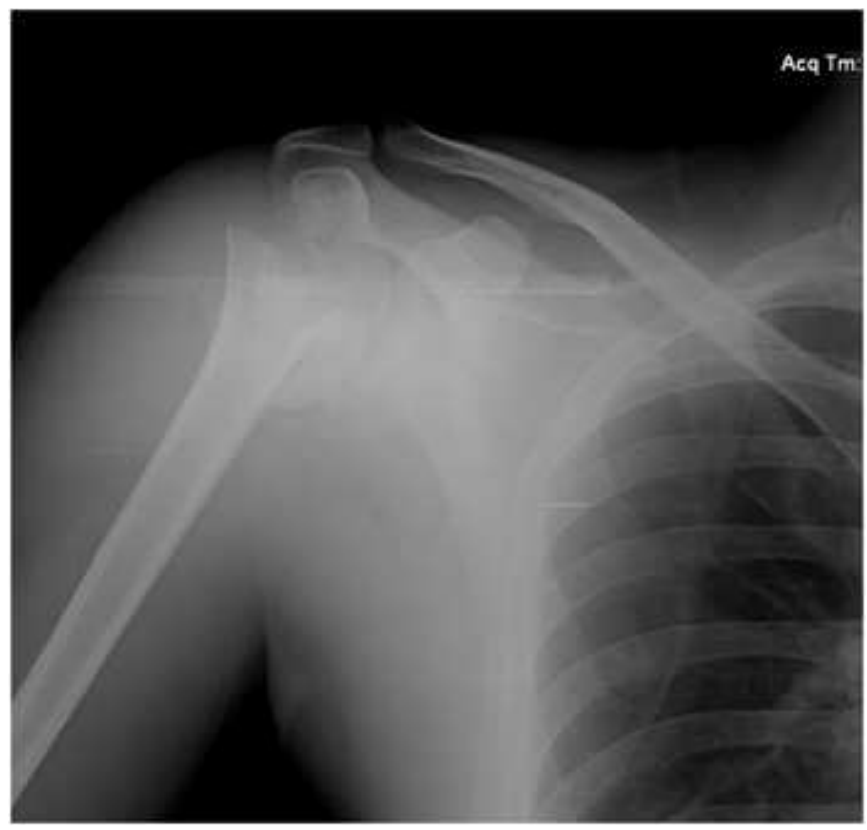

Fig 1b. Comminuted fracture anatomical neck of the right humerous and the posterior dislocation of the shoulder. 